\title{
Surface force analysis of glycine adsorption on different crystal surfaces of titanium dioxide $\left(\mathrm{TiO}_{2}\right)$
}

Narangerel Ganbaatar ${ }^{1,2}$, Kanae Imai ${ }^{2}$, Taka-aki Yano ${ }^{1,2,3}$ and Masahiko Hara1,2,3*0

\begin{abstract}
Surface force analysis with atomic force microscope (AFM) in which a single amino acid residue was mounted on the tip apex of AFM probe was carried out for the first time at the molecular level on titanium dioxide $\left(\mathrm{TiO}_{2}\right)$ as a representative mineral surface for prebiotic chemical evolution reactions. The force analyses on surfaces with three different crystal orientations revealed that the $\mathrm{TiO}_{2}(110)$ surface has unique characteristics for adsorbing glycine molecules showing different features compared to those on $\mathrm{TiO}_{2}(001)$ and (100). To examine this difference, we investigated thermal desorption spectroscopy (TDS) and the interaction between the PEG cross-linker and the three $\mathrm{TiO}_{2}$ surfaces. Our data suggest that the different single crystal surfaces would provide different chemical evolution field for amino acid molecules.
\end{abstract}

Keywords: Atomic force microscopy (AFM), Titanium dioxide $\left(\mathrm{TiO}_{2}\right), \mathrm{Glycine}$, Chemical evolution, Origins of life

\section{Introduction}

Many organic molecules are chemically or physically adsorbed to mineral surfaces in aqueous solutions. The extent of adsorption is dependent on variables including mineral type, surface roughness, type of adsorbed organic molecule, and the nature of the solvent with respect to properties such as $\mathrm{pH}$, ionic strength, temperature, and the concentration of dissolved ions, which may act as mediators for binding $[1,2]$. Among all mineral surfaces, titanium dioxide $\left(\mathrm{TiO}_{2}\right)$ is well known for its ability to adsorb biomolecules. Understanding the interaction of biomolecules with $\mathrm{TiO}_{2}$ surfaces is important for many areas of modern technology, including photocatalysis, dye-sensitized solar cells, and biological implants used in dental and orthopedic applications [3, 4]. Regarding research on origins of life, the surfaces of this mineral have long been studied as possible catalysts for small organic compound reactions and considered as a host inorganic catalyst in prebiotic evolution studies.

\footnotetext{
*Correspondence: masahara@echem.titech.ac.jp

${ }^{1}$ Chemical Evolution Lab Unit, Earth-Life Science Institute (ELSI), Tokyo Institute of Technology, Tokyo, Japan

Full list of author information is available at the end of the article
}

Moreover, rutile $\mathrm{TiO}_{2}$ is one of the minerals that have been discovered as micro- or nanoparticles in pre-solar dust grains (also known as interplanetary dust particles) and is included in the "mineral evolution" hypothesis, which outlines 10 stages of near-surface mineral diversification since the formation of the Earth [5]. However, not much is known about how $\mathrm{TiO}_{2}$ surfaces interact with biomolecules at the molecular level. Understanding the fundamental mechanisms underlying the adhesion of biomolecules on $\mathrm{TiO}_{2}$ surfaces is essential to the identification and improvement of materials suitable for medical applications. Therefore, spectroscopic analyses are required for assessing these mechanisms.

Previous theoretical studies of the interaction of amino acids with $\mathrm{TiO}_{2}$ surfaces, which were based on the density functional theory (DFT), have contributed to understanding the main adsorption modes and are used as prototypes to study protein-surface interactions. Recently, Sowmiya and Senthilkumar used first-principle calculations to determine that anatase $\mathrm{TiO}_{2}$ crystals with more (001) facets adsorb proline, hydroxyproline, and glycine, i.e., major components of the collagen protein. According to their results, amino acids strongly bind to the anatase 
$\mathrm{TiO}_{2}(001)$ surface in the dissociative adsorption configuration mainly because of the covalent interaction between the oxygen atoms of the amino acid and the titanium atoms on the mineral's surface [6]. Tillotson et al. used DFT calculations to study how small organic molecules interact with the $\mathrm{TiO}_{2}$ (110) surface. According to their calculations, glycine strongly binds to the Ti $5 f$ ion either via the amino $\left(-\mathrm{NH}_{2}\right)$ or the carboxyl group $(-\mathrm{COOH})$. However, the most stable binding mode for glycine occurred via the interaction of the two oxygen atoms of the carboxylic group oxygen with two Ti $5 f$ sites. The proposed reaction involves the transfer of a proton to the surface forming a hydroxyl group and is likely to also apply to larger molecules containing the $\mathrm{COOH}$ group [7]. Moreover, the DFT calculations of Tonner demonstrated a probable tilting stabilization effect between the surface-bound hydrogen atom and the nitrogen atom of the amino group, suggesting that van der Waals interactions stabilize the tilted configurations even further by reinforcing the attraction between the amino group and the surface [8].

Besides these theoretical studies, lots of experimental investigations have also been performed. Examples of these studies include the proline adsorption on $\mathrm{TiO}_{2}$ (110) and (011) single crystal surfaces that was demonstrated by synchrotron-based X-ray photoemission spectroscopy (XPS) and temperature programmed desorption (TPD) [9-11], and the adsorption of glycine on $\mathrm{TiO}_{2}$ (110) single crystals that was detected by synchrotronbased ultraviolet photoemission spectroscopy (UPS) $[12,13]$, scanning tunneling microscopy (STM) [14], and plane-wave DFT calculations [15].

However, as far as we know, there are no force studies of the adsorption of amino acids on the $\mathrm{TiO}_{2}$ surface at the molecular level. Therefore, we decided to study the adsorption of glycine, which is the amino acid with the simplest structure, on $\mathrm{TiO}_{2}$ surfaces by performing AFM force measurements.

\subsection{Surface structures of $\mathrm{TiO}_{2}(110),(100)$, and (001) for surface force analysis}

$\mathrm{TiO}_{2}$ is typically thought of as being chemically inert and as the most active photocatalyst found in nature, using the energy of light to promote chemical reactions. Importantly, $\mathrm{TiO}_{2}$ is an active catalyst for carbon-carbon bond formation reactions in which higher-molecular-weight compounds are synthesized from smaller ones [16].

$\mathrm{TiO}_{2}$ crystallizes in three major different structures: rutile (tetragonal, $\mathrm{a}=\mathrm{b}=4.584 \AA, \mathrm{c}=2.958 \AA$ ), anatase (tetragonal, $\mathrm{a}=\mathrm{b}=3.782 \AA, \mathrm{c}=9.514 \AA$ ), and brookite (rhombohedrical, $\mathrm{a}=5.436 \AA, \mathrm{b}=9.166 \AA, \mathrm{c}=5.135 \AA$ ) [16].

The rutile $\mathrm{TiO}_{2}$ (110) is known to be one of the most stable $\mathrm{TiO}_{2}$ crystal faces, while rutile and anatase are used in $\mathrm{TiO}_{2}$ applications and are of interest for surface science studies (Fig. 1a-c). Its surface contains two different kinds of titanium atoms. Along the (001) direction, rows of sixfold-coordinated $\mathrm{Ti}$ atoms alternate with fivefold-coordinated $\mathrm{Ti}$ atoms with one dangling bond that is perpendicular to the surface. Two kinds of oxygen atoms are created when the bridging oxygen atoms miss one bond to the $\mathrm{Ti}$ atom in the removed layer and are twofold coordinated [16]. These bridging oxygen atoms and the precise structure of the surface have been the subject of debate for many years. Because of their coordinative undersaturation, atoms from these rows are thought to be removed relatively easily by thermal annealing. The resulting point defects affect the overall chemistry of the surface [17].

On the (001) surface, all Ti atoms are fourfold-coordinated and all $\mathrm{O}$ atoms twofold-coordinated. Hence, it has been proposed that the number of broken bonds on this surface is higher than on the other low-index rutile surfaces discussed so far. Consequently, the (001) surface has a high surface energy and tends to facet or reconstruct.

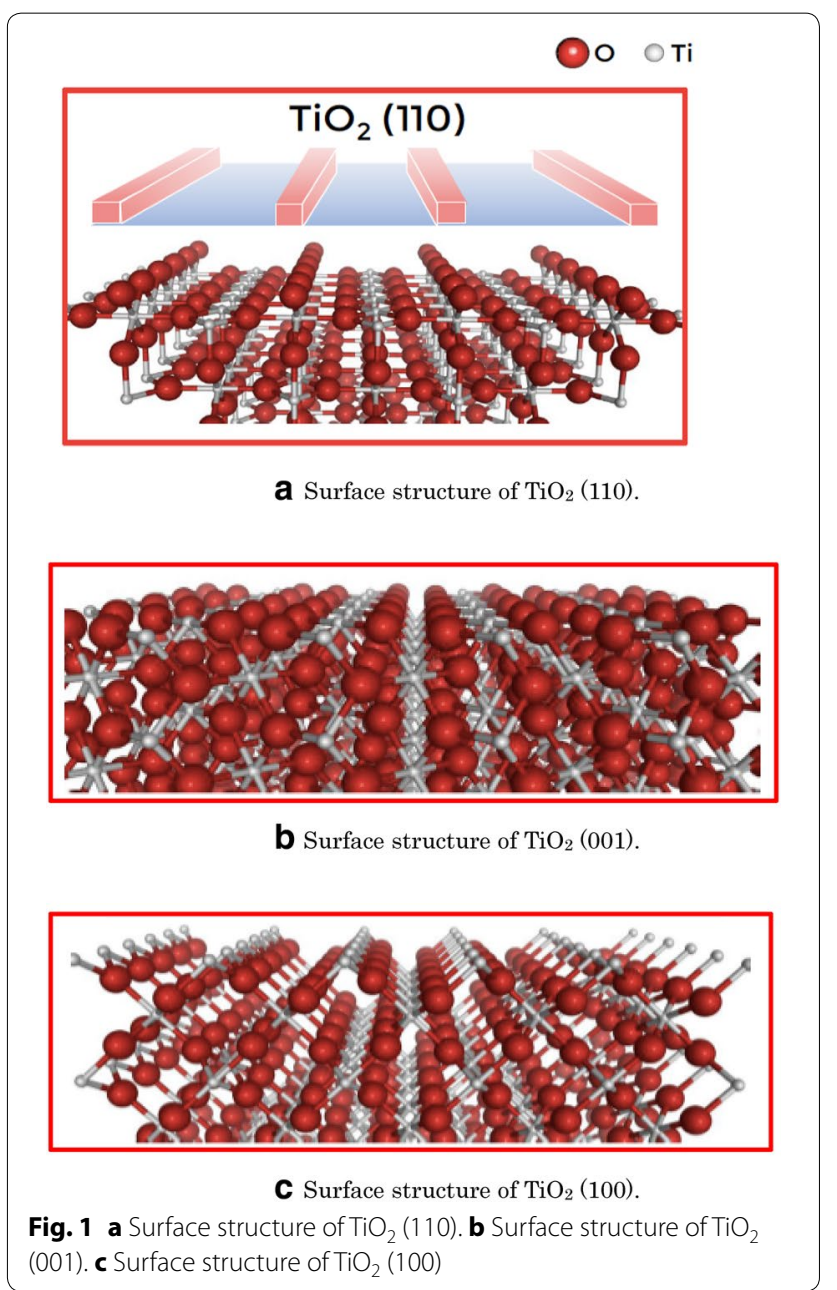


This surface is the least studied with respect to structural information [16].

The rutile $\mathrm{TiO}_{2}$ (100) surface has received considerably less attention than the (110) crystal face. Some research groups have conducted several theoretical calculations for this crystal surface. In general, all these calculations and theoretical approaches agree that $\mathrm{Ti}$ atoms are fivefold-coordinated on the (100) face.

Perron et al. carried out calculations on the different crystal structures of rutile $\mathrm{TiO}_{2}$ surfaces and computed rutile phases, structures, relaxations, and surface energies of the (110), (100), (101), and (001) faces. They found the (110) face to be the most stable one, followed by (100) and $(101)[18,19]$. Additional theoretical and experimental work aimed at resolving the geometry of this surface would be quite valuable.

\section{Experimental procedures}

\subsection{Sample preparations $\left(\mathrm{TiO}_{2}\right.$ substrates)}

The (110), (100), and (001) $\mathrm{TiO}_{2}$ surfaces were used to study the interactions with glycine. Commercially available rutile $\mathrm{TiO}_{2}$ substrates of all crystal planes were bought from Shinkosha (Tokyo, Japan).

New substrates were not cleaned by any further treatment and were directly used for AFM measurements. Used substrates were cleaned with deionized water and ethanol in ultrasonication to remove any contaminants from their surface.

\subsection{Attaching glycine molecule to the AFM cantilever tip} Glycine $\left(\mathrm{NH}_{2} \mathrm{CH}_{2} \mathrm{COOH}\right)$ molecules were anchored on an AFM tip apex to study the interactions of this amino acid with $\mathrm{TiO}_{2}$ (110), (100), and (001) surfaces. This linear molecule consists of three groups and two configurations are possible, as it is neutral in gas and zwitterionic in solid phases.

Moreover, glycine is the smallest of the amino acids. It is ambivalent, meaning that it can be inside or outside of the protein molecule. In aqueous solution and at, or near, neutral $\mathrm{pH}$, glycine exists predominantly in the zwitterion form.

With a well-established tip-modification method as previously reported [20], glycine molecules were introduced to the AFM tips. Commercial soft cantilevers (OMCL-TR400 PB-1, with both sides gold-coated) with nominal spring constants of $0.02 \mathrm{~N} / \mathrm{m}$ were used. Cantilevers were cleaned in UV ozone cleaner and followed by washing with ethanol and deionized water to be ready for the further modification steps. The cantilevers were first introduced in a mixture of two types of thiol reagents in ethanol (2 mM 1,8-octanedithiol and $20 \mathrm{mM}$ 6-mercapto-1-hexanol). Mixed solutions were used to increase the probability of single-molecule events by controlling the densities of molecules on the tips as well as to prevent aggregation on the tip surfaces. The cantilevers were inserted into the solution for more than $18 \mathrm{~h}$ to introduce the thiol groups. The modified cantilevers with thiol groups were washed with ethanol. MAL-PEGNHS cross-linker molecules were anchored to the tips by stable covalent bonds formed during the reaction of their maleimide ends with the $-\mathrm{SH}$ groups of alkanethiols. The thiol activated cantilevers were incubated for $60 \mathrm{~min}$ with $1 \mathrm{mg} / \mathrm{ml}$ of NHS-PEG-MAL in toluene. They were then washed several times with PBS to remove unreacted crosslinkers. Finally, glycine residues were cross-linked to the tips through the free NHS ends of the PEG crosslinker molecules by inserting the cantilevers into the solution for $2 \mathrm{~h}$.

\section{Results}

\subsection{AFM force measurements}

In the single-molecule force spectroscopy measurements, several hundred force-distance (F-D) curves were recorded with at least two glycine-modified AFM tips at room temperature in a phosphate-buffered saline (PBS) solution ( $\mathrm{pH} 7.4)$ to minimize the nonspecific interactions between the probe and the substrate. By lowering each modified AFM tip onto the measured surface to a maximum load of $400 \mathrm{pN}$ at a loading rate of $6 \mathrm{nN} / \mathrm{s}$, typical F-D curves were obtained from the contact between each tip and the three different crystal faces of $\mathrm{TiO}_{2}$ surfaces.

Glycine showed a specific interaction with the $\mathrm{TiO}_{2}$ (110) surface (Fig. 2a). The extension length (i.e., the length at the maximum physically possible extension of the cross-linker polymer) ranges from $\sim 10$ to $\sim 20 \mathrm{~nm}$. This range is considerable given the total length of molecules that were used to mount the glycine molecules on the tips. However, the fact that molecules can exist in different conformations may have contributed to the overall variety of the extension lengths. The cross-linker, NHSPEG-MAL, has a standard extended length of $\sim 10 \mathrm{~nm}$ while the preceding short thiol molecules are $\sim 2 \mathrm{~nm}$ long. Since the molecular length of the glycine is very small, most of the extension must have resulted from the linker molecules, which could have been extended to a total length of approximately $12-15 \mathrm{~nm}$. In addition, the adhesion forces recorded in these experiments ranged from $\sim 90$ to $150 \mathrm{pN}$ and the probability of adhesion was low $(8 \sim 10 \%)$.

A histogram was constructed for the number of adhesion events recorded on $\mathrm{TiO}_{2}$ (110) surface (total number of events: $\mathrm{n} \approx 60$ ) versus rupture force and was used to obtain the most probable force (MPF), which was $128.31 \pm 38.88 \mathrm{pN}$ (Fig. $2 \mathrm{~b}$ ). Only the rupture events that 


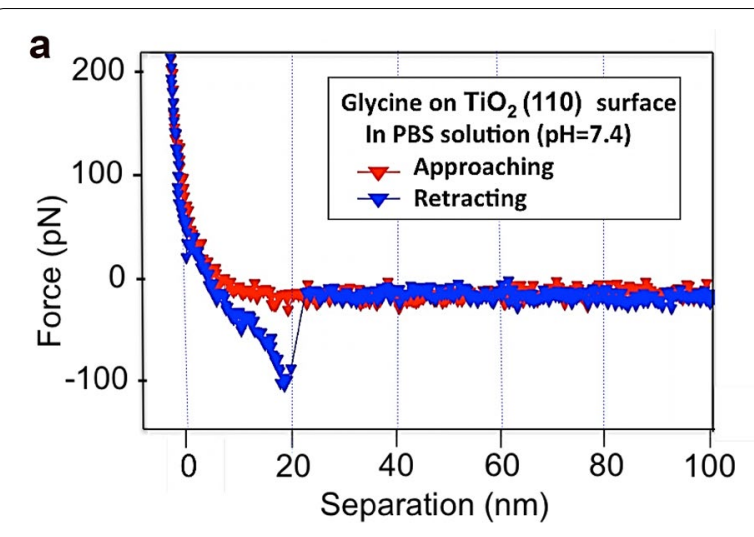

b

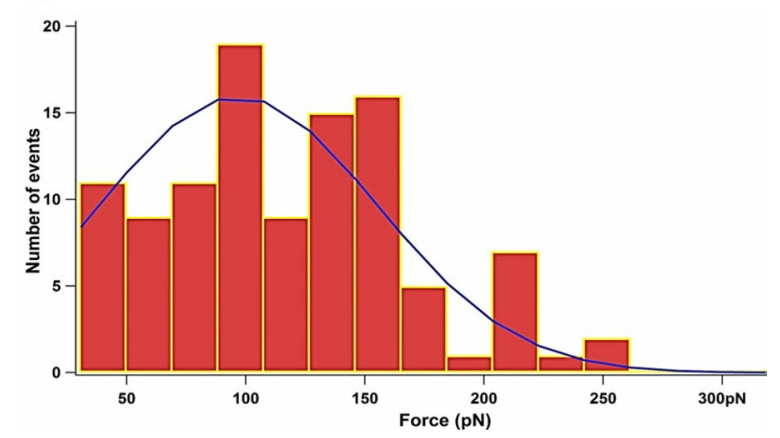

Fig. 2 a Specific adhesion curve obtained on $\mathrm{TiO}_{2}(110)$ surface. $\mathbf{b}$ Unbinding force histogram. MPF for glycine on $\mathrm{TiO}_{2}(110)$ surface at a loading rate of $6 \mathrm{nN} / \mathrm{s}$ (pH 7.4). Fitting was performed with a Gaussian fit and shows a mean force of $128.31 \pm 38.88 \mathrm{pN}$ occurred when the PEG linkers were extended by $\sim 10$ to $\sim 20 \mathrm{~nm}$ are shown in the given histograms.

Specific adhesion was also observed in the force measurements of glycine on $\mathrm{TiO}_{2}$ (100) surface (Fig. 3a). The extension length was considerable and ranged from $\sim 10$ to $\sim 20 \mathrm{~nm}$. The adhesion forces recorded in these experiments ranged from $\sim 60$ to $120 \mathrm{pN}$, i.e., weaker than those recorded on the $\mathrm{TiO}_{2}(110)$ surface.

The histogram was built by collecting the appropriate force curves (total number of events: $\mathrm{n} \approx 60$ ) and the Gaussian fit indicated an MPF of $96.24 \pm 34.22 \mathrm{pN}$ (Fig. 3b). Only those rupture events that occurred when the PEG linkers were extended by $\sim 10$ to $\sim 20 \mathrm{~nm}$ are shown in the histogram.

Glycine also showed a specific interaction with the $\mathrm{TiO}_{2}$ (001) surface (Fig. 4a). There was no significant difference in adhesion forces between glycine and the $\mathrm{TiO}_{2}$ (001) surface compared to the $\mathrm{TiO}_{2}(100)$ surface. Moreover, the resulting histogram indicated a similar MPF value $(92.85 \pm 33.72 \mathrm{pN})$ (Fig. 4b). The adhesion force was higher on the $\mathrm{TiO}_{2}(110)$ surface $(128.31 \pm 38.88 \mathrm{pN})$, compared to the other two surfaces.

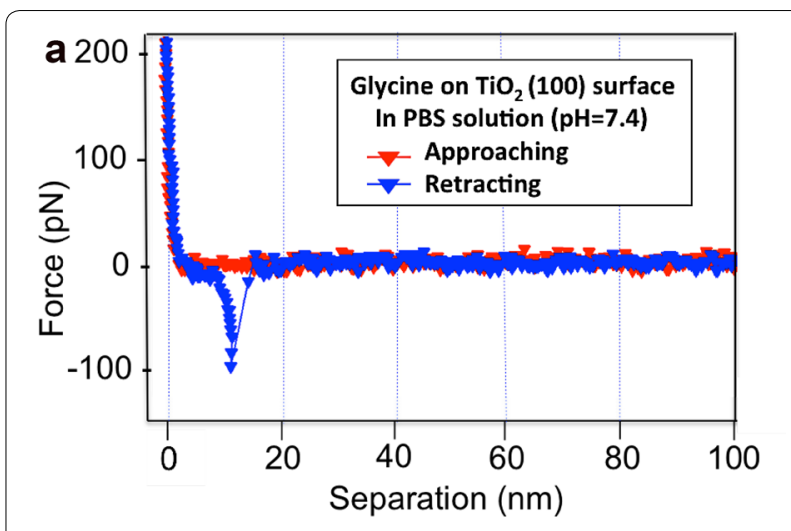

b

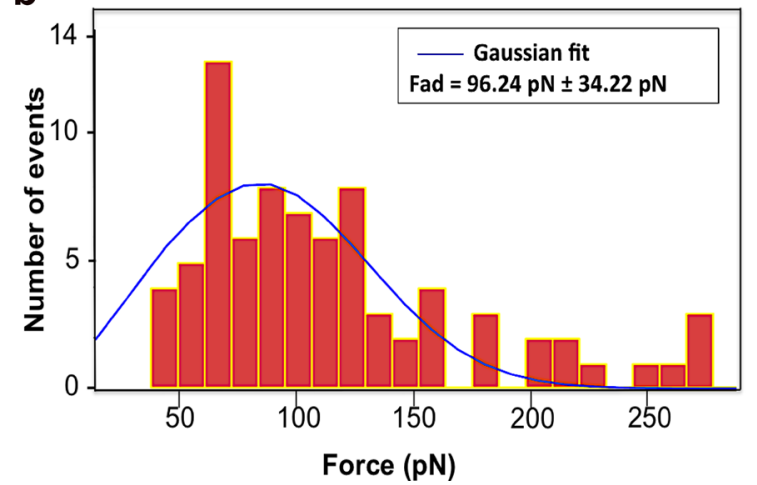

Fig. 3 a Specific adhesion curve obtained on $\mathrm{TiO}_{2}$ (100) surface. b Unbinding force histogram. MPF for glycine on $\mathrm{TiO}_{2}(100)$ surface at a loading rate of $6 \mathrm{nN} / \mathrm{s}$ ( $\mathrm{pH}$ 7.4). Fitting was performed with a Gaussian fit and showed a mean force of $96.24 \pm 34.22 \mathrm{pN}$

\subsection{Thermal desorption spectroscopy (TDS) measurements} Thermal Desorption Spectroscopy (TDS) measurements were conducted to check the glycine desorption on all three $\mathrm{TiO}_{2}$ (110), (100), and (001) crystal plane surfaces. All surfaces displayed a desorption peak at $\mathrm{m} / \mathrm{z} 30$ indicating the desorption of glycine monomers. With respect to temperature, desorption at $\mathrm{m} / \mathrm{z} 30$ had sharp peaks at $260-285{ }^{\circ} \mathrm{C}$ in the case of $\mathrm{TiO}_{2}(110)$, at $240{ }^{\circ} \mathrm{C}$ in $\mathrm{TiO}_{2}$ (100), and at $240{ }^{\circ} \mathrm{C}$ in $\mathrm{TiO}_{2}$ (001) (Fig. 5a). These results suggest that, at a higher temperature, $\mathrm{TiO}_{2}(110)$ interacts with glycine more strongly than with other crystal faces. This is consistent with the AFM force measurements in which the adhesion force was higher for the $\mathrm{TiO}_{2}(110)$ surface compared to the other two surfaces.

TDS measurements were also carried out on mica and pyrite for comparison with $\mathrm{TiO}_{2}$ (Fig. 5b). The maximum desorption temperature for the glycine monomer $(\mathrm{m} / \mathrm{z} 30)$ was $200{ }^{\circ} \mathrm{C}$ for $\mathrm{TiO}_{2}$ and around $150{ }^{\circ} \mathrm{C}$ for mica and pyrite. Importantly, $\mathrm{TiO}_{2}$ also displayed desorption peaks at $\mathrm{m} / \mathrm{z} 71$ and $\mathrm{m} / \mathrm{z} 114$, corresponding to glycine dimers with a maximum at $245^{\circ} \mathrm{C}$. The fact that, among 

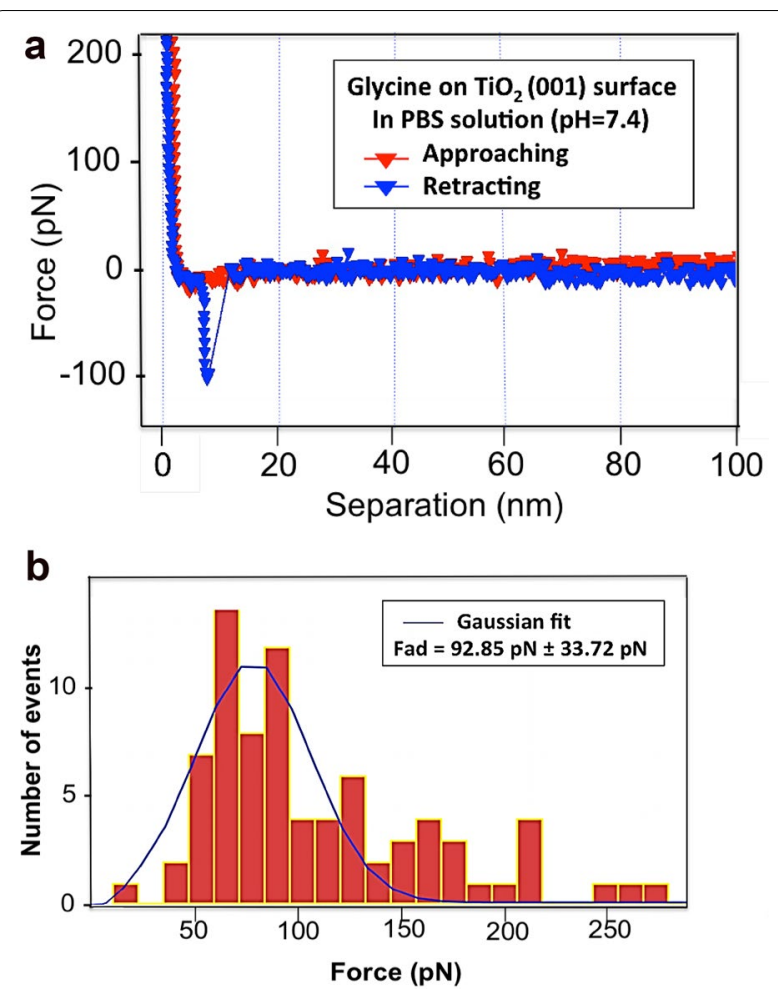

Fig. 4 a Specific adhesion curve obtained on $\mathrm{TiO}_{2}(001)$ surface. $\mathbf{b}$ Unbinding force histogram. MPF for glycine on $\mathrm{TiO}_{2}(001)$ surface at a loading rate of $6 \mathrm{nN} / \mathrm{s}$ ( $\mathrm{pH}$ 7.4). Fitting was performed using a Gaussian fit and showed a mean force of $92.85 \pm 33.72 \mathrm{pN}$

the three kinds of surfaces, polymerization of glycine was only observed on $\mathrm{TiO}_{2}$, suggests that $\mathrm{TiO}_{2}$ plays an important role in the polymerization reaction of glycine.

Overall, the TDS results revealed the factors because of which glycine interacts more strongly with $\mathrm{TiO}_{2}$ compared to the other minerals. Interaction is mainly categorized into two types: physical and chemical adsorption. Physical adsorption is weak and results from van der Waals forces or electrical interactions. Chemical adsorption is strong as electron exchange takes place between the adsorbed molecule and the surface, resulting in the formation of bonds (covalent, ionic, metal, or coordinate). These results were in agreement with the force measurements in which glycine showed specific interaction with $\mathrm{TiO}_{2}$ but not pyrite or mica surfaces.

\section{Discussion}

\subsection{Difference in force curves}

Even though all $\mathrm{TiO}_{2}$ crystal surfaces showed specific adhesion towards the glycine-modified AFM tips, differences were observed regarding the shape of the force curves. There was not a significant difference between the F-D curves obtained on $\mathrm{TiO}_{2}(100)$ and $\mathrm{TiO}_{2}(001)$ surfaces, but the shape of adhesion curves at $\mathrm{TiO}_{2}(110)$ was very different compared to the $\mathrm{F}-\mathrm{D}$ curves obtained at the other two surfaces (Fig. 6a). In the case of $\mathrm{TiO}_{2}(110)$, the attraction began after the tip and the surface had already been in contact, resulting in a force curve with a broader shape. It should be noted that the same modified AFM tips were used on all three substrates, while the substrates were measured in the following order: $\mathrm{TiO}_{2}$ (110), (100), and (001). Thus, the difference in the shape of the curves cannot be attributed to contamination. We made the hypothesis that an interaction between the PEG linker and the surface taking place before the adhesion of the glycine molecules to the $\mathrm{TiO}_{2}$ (110) surface may be responsible for the difference in curve shapes. To test this hypothesis, we conducted experiments to check the interaction between the PEG molecules and the $\mathrm{TiO}_{2}$ surfaces.

\subsection{Possible interaction between the PEG linker and $\mathrm{TiO}_{2}$ surfaces}

In order to explain the difference between the force curves, we conducted further experiments to check the interaction between the PEG cross-linker and $\mathrm{TiO}_{2}$ (110), (100), and (001) substrates. The tip modification process was repeated without the last step, i.e., without the attachment of amino acid molecules. In other words, the AFM tip modification was stopped after the attachment of the PEG cross-linker (Fig. 7a). Measurements were performed in PBS (pH 7.4) solution under the exact same conditions (loading rate, loading force, etc.) of the glycine experiment.

Force measurements did not reveal any specific interaction of PEG-modified tips with $\mathrm{TiO}_{2}(100)$ and (001) surfaces. Measurements on the $\mathrm{TiO}_{2}$ (100) surface were dominated by force curves displaying no interaction (Fig. 7b), while some curves showing non-specific interaction were also observed. When we changed the substrate to $\mathrm{TiO}_{2}$ (001), only force curves showing nonspecific interactions caused by electrostatic forces were obtained (Fig. 7c).

Interestingly, a different pattern of interaction was obtained in the case of the $\mathrm{TiO}_{2}$ (110) surface. We observed a plateau in the force measurements between the PEG-modified tip and the $\mathrm{TiO}_{2}(110)$ surface (Fig. 8a, b). This plateau represents a weak physical adsorption of polymer molecules or binding with the surface via the formation of ionic bonds. The length of the plateau directly reflects the length of the adsorbed polymer, whereas its height corresponds to the desorption force that is required to desorb one or multiple polymers from the opposite surface [16].

The average plateau length observed in this experiment was around $20 \mathrm{~nm}$, which corresponds to a considerable extension of the PEG molecule. However, we cannot be certain whether one or multiple polymer chains are 


\section{a}

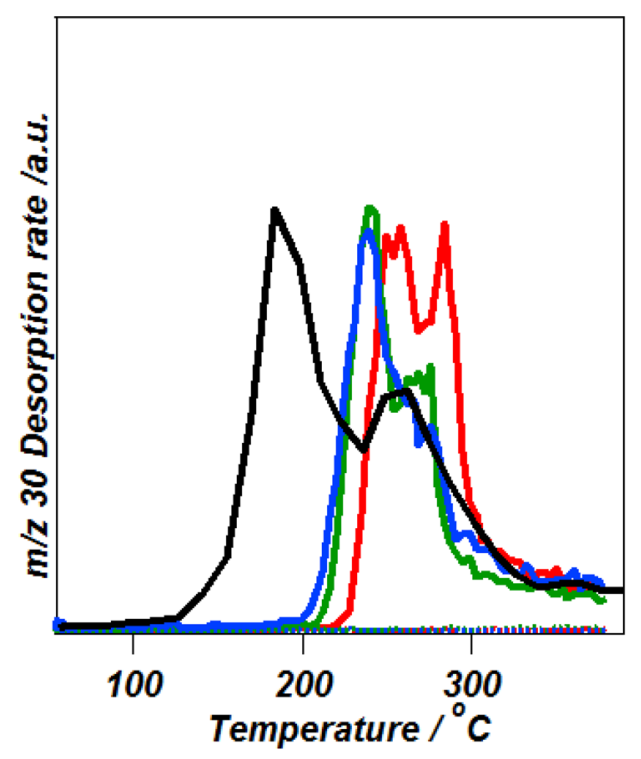

b

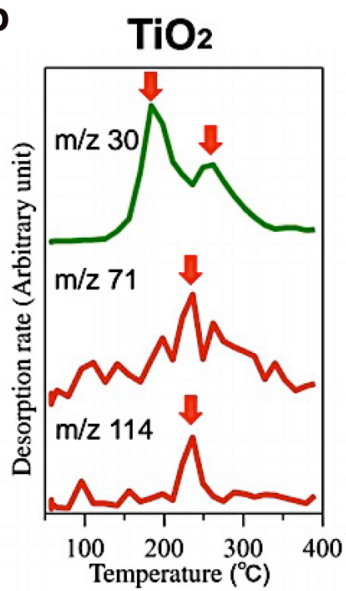

Mica

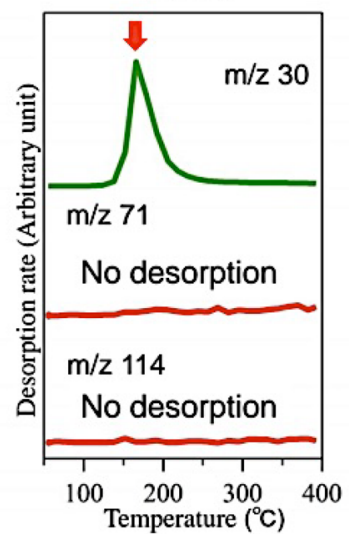

Pyrite

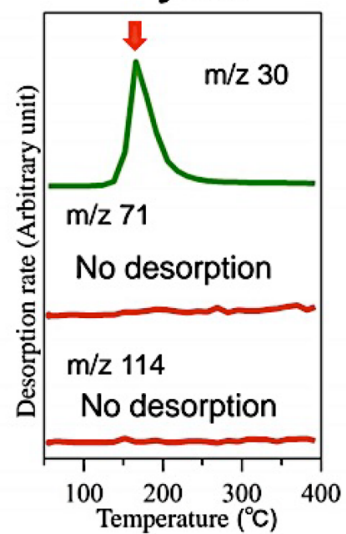

Fig. 5 a Desorption of glycine from single crystals of $\mathrm{TiO}_{2}$ (110), (100), and (001). Polycrystalline was included as a reference substrate. Red line $=\mathrm{TiO}_{2}(110)$, green line $=\mathrm{TiO}_{2}(100)$, blue line $=\mathrm{TiO}_{2}(001)$, and black line = polycrystalline. $\mathbf{b}$ Comparison of interactions between glycine and $\mathrm{TiO}_{2}$, mica, or pyrite by TDS measurements

involved in this plateau feature. Plateau force curves in AFM studies have no clear interpretation, thus it is difficult to conclude whether we are dealing with a single polymer chain interaction. In rare occasions, force curves displaying some overstretching and step-like plateaus were obtained. These steps represent the desorption of individual molecules of different lengths. Such weak long-range attraction indicates that some kind of contact is maintained over distances exceeding the standard length of the PEG molecule.

Most importantly, this plateau feature was only detected when measuring the $\mathrm{TiO}_{2}$ (110) surface, implying that it has unique features of adsorption towards glycine molecules compared to $\mathrm{TiO}_{2}(001)$ and (100) surfaces. Our force measurement result was indeed correlated with the difference that was observed between the force curves of glycine on different $\mathrm{TiO}_{2}$ surfaces.

As a reference, we performed force measurements on the $\mathrm{TiO}_{2}$ surfaces using tips that had only undergone the first step of modification (i.e., they had only been modified with the thiol mixture). Only force curves showing molecular aggregation or non-specific interactions due to van der Waals attraction forces were obtained. This proves that the plateau force of interaction indeed originated from the molecules on the tip and the substrate. 


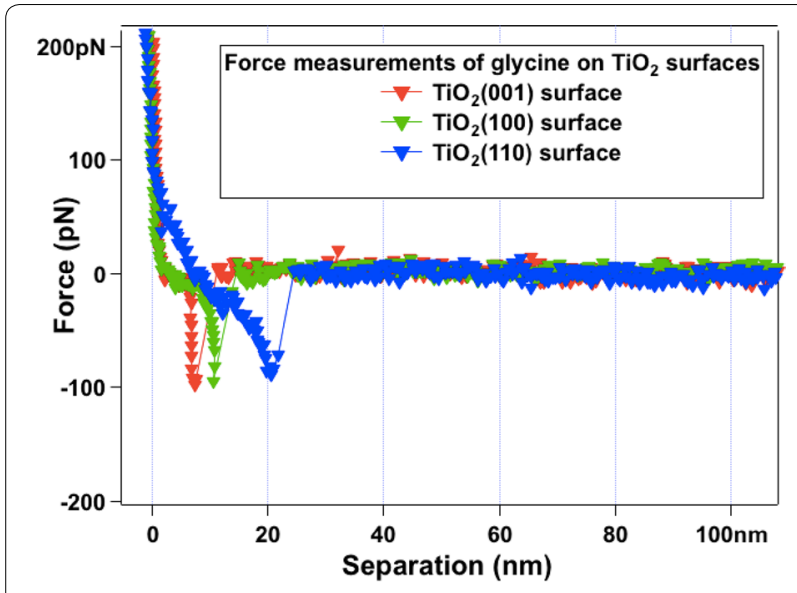

Fig. 6 Force measurements between a glycine-modified tip and $\mathrm{TiO}_{2}$ (110), (100), and (001) surfaces

\subsection{Glycine adsorption mode on $\mathrm{TiO}_{2}(110)$ surface}

There are plenty of previous studies about glycine adsorption on $\mathrm{TiO}_{2}$ crystals. Most of them used theoretical approaches to determine the binding model of glycine on $\mathrm{TiO}_{2}$. According to literature based on DFT molecular dynamics simulations, glycine is adsorbed on $\mathrm{TiO}_{2}$ via its zwitterion form, preferentially via deprotonation of the carboxyl group, and forms a $(2 \times 1)$ overlayer [3]. Ojamäe et al. suggested a binding model in which the adsorbate binds to surface titan atoms through its carboxylic end (by the formation of an oxygen bridge) and to a surface oxygen ion through its ammonium end (by an $\mathrm{H}$-bond).

In this model, hydrogen bonding via the amine group leads to the further stabilization of the adsorption. They conclude that even though the bridge and monodentate modes are highly favorable, all of these binding modes can contribute to the glycine adsorption on the $\mathrm{TiO}_{2}$ (110) surface. The bidentate modes, where the two oxygen atoms in the carboxylic group attach directly to the fivefold-coordinated Ti sites at the surface, are analogous to the most favorable adsorption mode of formic acid described above. Glycine can also form hydrogen bonds with the surface via the amino group, providing additional stability and leading to a tilting of the molecule with respect to its "vertical" configuration [6].

Moreover, Hazen et al. reported that the $\mathrm{TiO}_{2}$ (110) surface consists of steps at the atomic scale. The remarkably uniform pattern of steps has an orientation that provides under-coordinated $\mathrm{Ti}$ atoms to adsorb amino acids. They strongly suggested that amino acids attach at these a

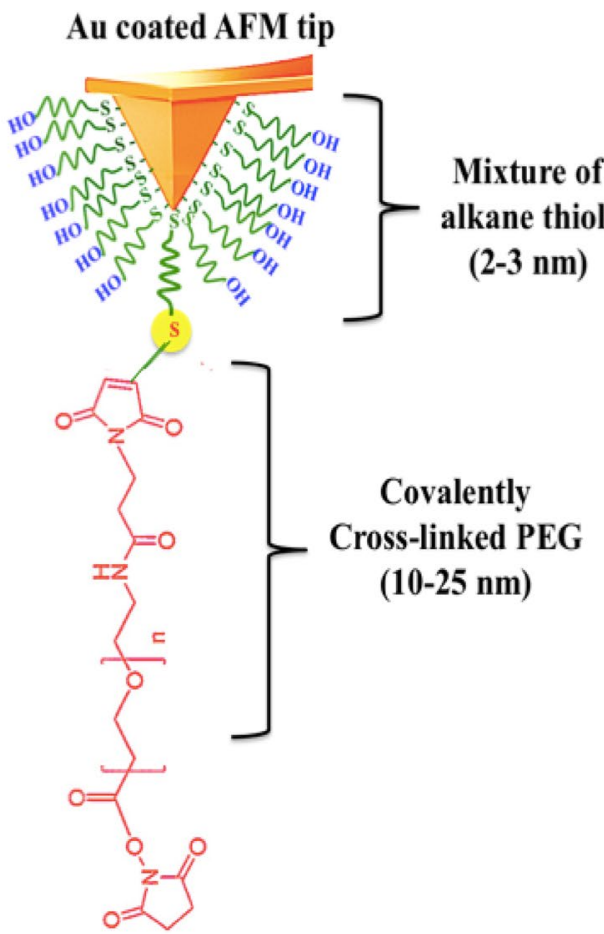

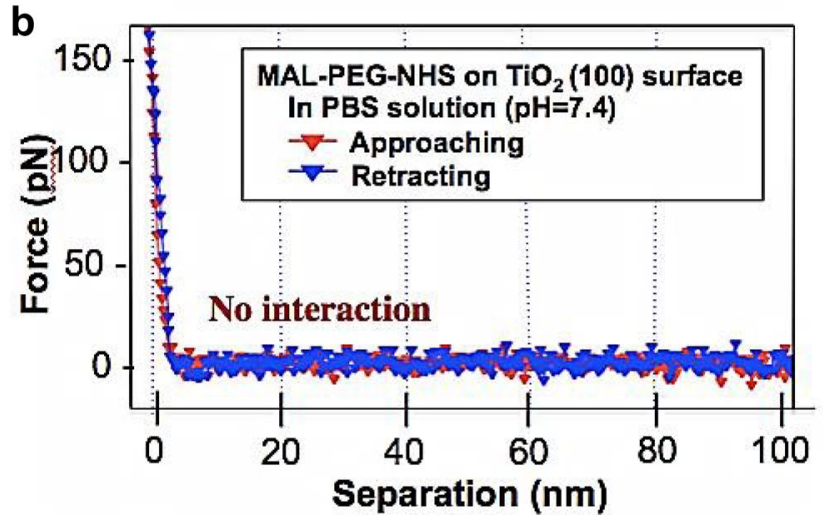

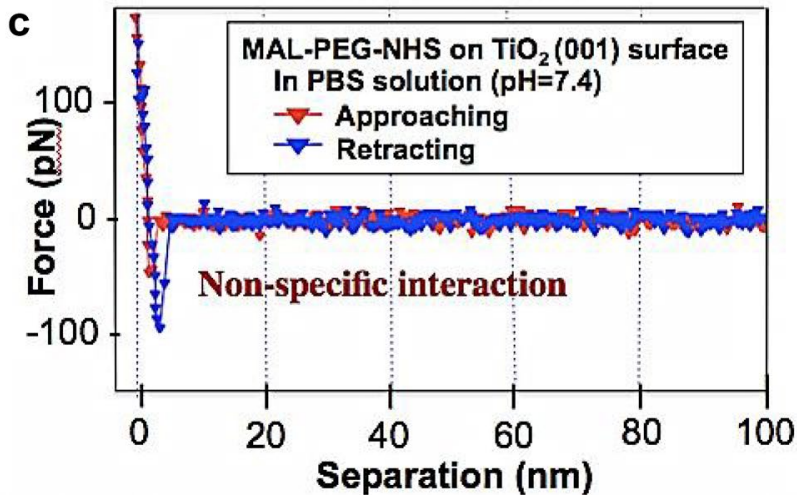

Fig. 7 (a) Schematic presentation of the modified AFM tip that was used in this experiment; Representative force curves of PEG-modified tip (b) on $\mathrm{TiO}_{2}(100)$ and $(\mathbf{c})$ on $\mathrm{TiO}_{2}(001)$ surface 


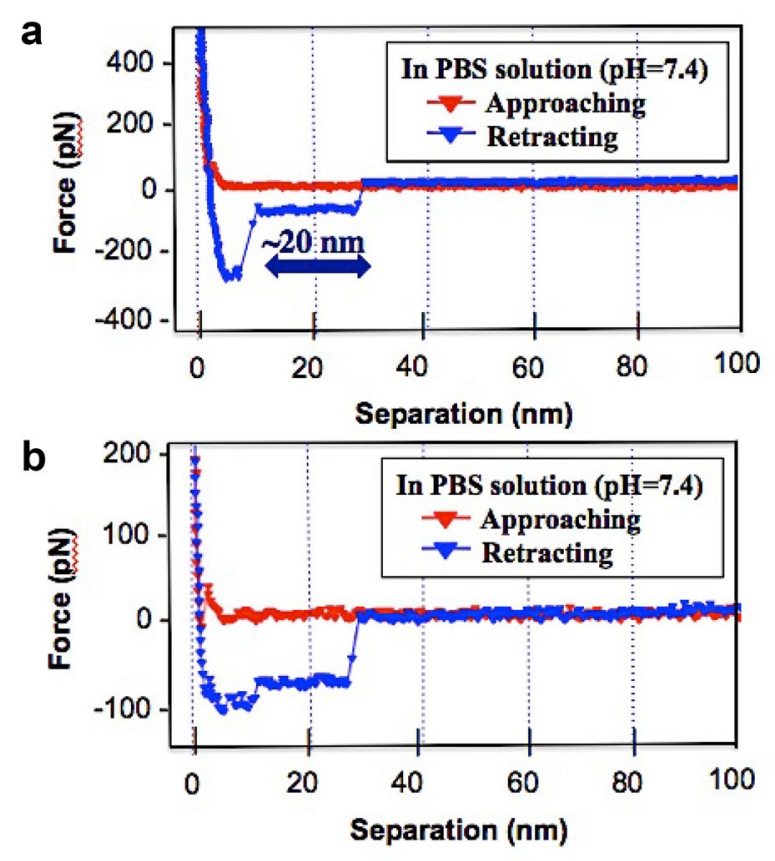

Fig. 8 a, b Representative force curves between PEG-modified tips and the $\mathrm{TiO}_{2}(110)$ surface

steps on the (110) surfaces of rutile. It is known that, in all crystals, steps present special bonding environments for adsorbing molecules.

\section{Conclusions}

In this work, we studied the interaction between glycine and three different $\mathrm{TiO}_{2}$ crystal surfaces for the first time at the molecular level using AFM force techniques. The adsorption of glycine on $\mathrm{TiO}_{2}$ was observed by AFM force analysis in which all three crystals $\left[\mathrm{TiO}_{2}(110)\right.$, (001), and (100)] used in this study showed specific interactions with the glycine-modified AFM tips, whereas no adhesion was observed on pyrite. The surface force results were in good agreement with the TDS measurements in which glycine showed a desorption peak on the $\mathrm{TiO}_{2}$ surfaces, whereas no desorption was observed on mica and pyrite.

The difference in adsorption on the $\mathrm{TiO}_{2}$ surfaces was attributed to the shape of force curves on the $\mathrm{TiO}_{2}(110)$ surface. The adhesion forces and the shapes of the force curves on $\mathrm{TiO}_{2}(001)$ and (100) surfaces were similar. However, the $\mathrm{TiO}_{2}$ (110) surface showed a different feature of adhesion in both the AFM force results as well as in the TDS measurements. We assume that the PEG cross-linker may interact with the $\mathrm{TiO}_{2}$ (110) surface before the glycine adsorption. Therefore, the crystal orientation of $\mathrm{TiO}_{2}$ may influence the amino acid adsorption pathway; however, how the surface structure of this crystal attaches the organic molecules is still under investigation.

As outlined in the introduction, the biomolecule adsorption on $\mathrm{TiO}_{2}$ surfaces is also studied for use in many technological and medical applications. In origins of life studies, a fundamental level of understanding of the role of minerals in chemical evolution can be achieved by studying how molecules interact with $\mathrm{TiO}_{2}$ surfaces in a way similar to that applied to other candidate catalysts. Our results may contribute to the chemical evolution studies by adding to the knowledge of how biomolecules interact with the $\mathrm{TiO}_{2}$ surface, which is considered as one of the host inorganic catalysts in prebiotic evolution studies. Moreover, knowledge of these interactions might also facilitate the use of $\mathrm{TiO}_{2}$ in biotechnological applications.

\section{Authors' contributions}

NG carried out the AFM studies and draft manuscript writing. KI assisted the crystal plane dependence by TDS. TY and MH supervised the research. All authors read and approved the manuscript.

\section{Author details}

${ }^{1}$ Chemical Evolution Lab Unit, Earth-Life Science Institute (ELSI), Tokyo Institute of Technology, Tokyo, Japan. ${ }^{2}$ Department of Electronic Chemistry, Interdisciplinary Graduate School of Science and Engineering, Tokyo Institute of Technology, Yokohama, Japan. ${ }^{3}$ Department of Chemical Science and Engineering, School of Materials and Chemical Technology, Tokyo Institute of Technology, Yokohama, Japan.

\section{Competing interests}

The authors declare that they have no competing interests.

\section{Funding}

This work was supported by JSPS KAKENHI Grant-in-Aid for Scientific Research on Innovative Areas "Hadean Bioscience", Grant Number JP26106003.

\section{Publisher's Note}

Springer Nature remains neutral with regard to jurisdictional claims in published maps and institutional affiliations.

Received: 10 September 2017 Accepted: 1 November 2017

Published online: 08 December 2017

\section{References}

1. W. Stumm, Chemistry of the solid-water interface: processes at the mineralwater and particle-water interface in natural systems (Wiley, New York, 1992)

2. D.A. Sverjensky, C.M. Jonsson et al., Glutamate surface speciation on amorphous titanium dioxide and hydrous ferric oxide. Environ. Sci. Technol. 42(16), 6034-6039 (2008)

3. T.L. Thompson, J.T. Yates, Surface science studies of the photoactivation of $\mathrm{TiO}_{2}$-new photochemical processes. Chem. Rev. 106, 4428-4453 (2006)

4. A.G. Thomas, K.L. Syres, Adsorption of organic molecules on rutile $\mathrm{TiO}_{2}$ and anatase $\mathrm{TiO}_{2}$ single crystal surfaces. Chem. Soc. Rev. $\mathbf{4 1}(11)$, 4207-4217 (2012)

5. James H. Cleaves II, Andrea Michalkova Scott et al., Mineral-organic interfacial processes: potential roles in the origins of life. Chem. Soc. Rev. 41, 5502-5525 (2012) 
6. M. Sowmiya, K. Senthilkumar, Adsorption of proline, hydroxyproline and glycine on anatase (001) surface: a first-principle study. Theor. Chem. Acc. 135, 12 (2016)

7. M. Tillotson, P. Brett, et al. Adsorption of organic molecules at the $\mathrm{TiO}_{2}$ (110) surface: the effect of van der Waals interactions. Surf. Sci. 632, 142-153 (2015)

8. R. Tonner, Adsorption of proline and glycine on the $\mathrm{TiO}_{2}(110)$ surface: a density functional theory study. Chem Phys Chem. 11, 1053-1061 (2010)

9. G.J. Fleming, H. Idriss, Probing the reaction pathways of DL-Proline on $\mathrm{TiO}_{2}$ (001) single crystal surfaces. Langmuir 20(18), 7540-7546 (2004)

10. G.J. Fleming, K. Adib et al., The adsorption and reactions of the amino acid proline on rutile $\mathrm{TiO}_{2}$ (110) surfaces. Surf. Sci. 602(12), 2029-2038 (2008)

11. G.J. Fleming, K. Adib et al., Proline adsorption on $\mathrm{TiO}_{2}$ (110) single crystal surface: a study by high resolution photoelectron spectroscopy. Surf. Sci. $\mathbf{6 0 1}(24), 5726-5731$ (2007)

12. E. Soria, I. Colera et al., A study of photon-induced processes with adsorption-desorption of glycine at the $\mathrm{TiO}_{2}(110)(1 \times 2)$ surface. Surf. Sci. 451(1-3), 188-196 (2000)

13. E. Soria, E. Roman et al., Observations with synchrotron radiation (20$120 \mathrm{eV}$ ) of the $\mathrm{TiO}_{2}$ (110)-glycine interface. Surf. Sci. 433, 543-548 (1999)
14. T.Z. Qiu, M.A. Barteau, STM study of glycine on $\mathrm{TiO}_{2}$ (110) single crystal surfaces. Journal of Colloid and Interface Science 303(1), 229-235 (2006)

15. L. Ojamae, C. Aulin, IR and quantum-chemical studies of carboxylic acid and glycine adsorption on rutile $\mathrm{TiO}_{2}$ nanoparticles. J. Colloid. Interface. Sci. 296(1), 71-78 (2006)

16. U. Diebold, The surface science of titanium dioxide. Surf. Sci. Rep. 48 , 53-229 (2003)

17. L. Ojamäe et al., IR and quantum-chemical studies of carboxylic acid and glycine adsorption on rutile $\mathrm{TiO}_{2}$ nanoparticles. J. Colloid. Interface. Sci. 296, 71-78 (2006)

18. Kenneth J.T. Livi, Bernhard Schaffer et al., Atomic-scale surface roughness of rutile and implications for organic molecule adsorption. Langmuir $\mathbf{2 9}$, 6876-6883 (2013)

19. H. Perron, C. Domain et al., Optimisation of accurate rutile TiO2 (110), (100), (101) and (001) surface models from periodic DFT calculations. Theor. Chem. Acc. 117, 565-574 (2007)

20. N. Ganbaatar, N. Matsuzaki, Y. Nakazawa, R. Afrin, M. Aono, T. Yano, T. Hayashi, M. Hara, Surface force analysis of pyrite (FeS2): its reactivity to amino acid adsorption. Adv. Mater. Phys Chem. 6, 167-176 (2016). https://doi.org/10.4236/ampc.2016.67018

\section{Submit your manuscript to a SpringerOpen ${ }^{\circ}$ journal and benefit from:}

- Convenient online submission

- Rigorous peer review

- Open access: articles freely available online

- High visibility within the field

- Retaining the copyright to your article

Submit your next manuscript at $\boldsymbol{\nabla}$ springeropen.com 